\title{
BMJ Open Use of interrupted time-series analysis to characterise antibiotic prescription fills across pregnancy: a Norwegian nationwide cohort study
}

\author{
Nhung Thi Hong Trinh (D) , ${ }^{1}$ Sarah Hjorth (D) , ${ }^{1}$ Hedvig Marie Egeland Nordeng ${ }^{1,2}$
}

To cite: Trinh NTH, Hjorth S, Nordeng HME. Use of interrupted time-series analysis to characterise antibiotic prescription fills across pregnancy: a Norwegian nationwide cohort study. BMJ Open 2021;11:e050569. doi:10.1136/ bmjopen-2021-050569

- Prepublication history and additional supplemental material for this paper are available online. To view these files, please visit the journal online (http://dx.doi.org/10.1136/ bmjopen-2021-050569).

Received 23 February 2021 Accepted 17 November 2021

D Check for updates

(c) Author(s) (or their employer(s)) 2021. Re-use permitted under CC BY-NC. No commercial re-use. See rights and permissions. Published by BMJ.

${ }^{1}$ PharmacoEpidemiology and Drug Safety Research Group, Department of Pharmacy, and PharmaTox Strategic Initiative, Faculty of Mathematics and Natural Sciences, University of Oslo, Oslo, Norway

${ }^{2}$ Department of Child Health and Development, Norwegian Institute of Public Health, Oslo, Norway

Correspondence to Dr Nhung Thi Hong Trinh; nthtrinh@farmasi.uio.no

\section{ABSTRACT}

Objectives Antibiotics are the most frequently prescribed medications for pregnant and breastfeeding women. We applied interrupted time-series analysis (ITSA) to describe antibiotic prescription fills patterns in pregnant women and examined recurrent antibiotic fills in subsequent pregnancies. Designs A population-based drug utilisation study.

Setting Norwegian primary care.

Participants 653058 pregnancies derived from Medical Birth Registry of Norway linked to the Norwegian Prescription Database (2006-2016).

Main outcome measure Proportion of pregnancies exposed to antibiotics aggregated by week in pregnancy time windows.

Statistical analyses We descriptively analysed antibiotic prescription fills patterns and components in pregnant women. The changes in antibiotic fills in pregnancy time windows were assessed using ITSA. Interruptions points at week 4 to week 7 into pregnancy and delivery were used. Factors associated with antibiotic fills during pregnancy were identified using generalised estimating equations for Poisson regression. Recurrent antibiotic use was estimated using proportion of women who filled antibiotic prescription in a subsequent pregnancy.

Results Antibiotics were filled in $27.6 \%$ pregnancies. The ITSA detected an immediate decrease of 0.07 percentage points $(95 \% \mathrm{Cl}-0.13$ to -0.01$)$ in the proportion of exposed pregnancies at 4 weeks after conception, mainly among women taking folic acid before pregnancy. This proportion increased shortly after delivery (immediate change $=1.61$ percentage points $(95 \% \mathrm{Cl} 0.31$ to 2.91)) then decreased gradually afterwards (change in slope $=-0.19$ percentage points, $95 \% \mathrm{Cl}-0.34$ to -0.05$)$ ). The strongest factor associated with antibiotic fills during pregnancy was having recurrent urinary tract infections (adjusted $\mathrm{OR}=2.65,95 \% \mathrm{Cl} 2.59$ to 2.72). Women who had filled antibiotics during a pregnancy were up to three times more likely to fill antibiotics in the subsequent pregnancies. Conclusions ITSA highlighted important impact of pregnancy and delivery on antibiotic fillings. Having antibiotic fills in a pregnancy was associated with recurrent antibiotic fills in subsequent ones.

\section{INTRODUCTION}

Antibiotics are the most frequently prescribed medications for pregnant and breastfeeding women ranging from $20 \%$ to $49 \% .^{1-8}$ In
Strengths and limitation of this study

- First study to use interrupted time-series analysis to examine the effect of pregnancy on antibiotic prescription filling patterns.

- First population-based study to follow-up women from the beginning of their reproductive history and quantify recurrent antibiotic filling pattern.

- Diagnoses associated with antibiotic prescription fills were not assessed.

- Appropriateness of antibiotic filling was not assessed.

- Antibiotic filling rate may not truly reflect prescribing and utilising rate.

outpatient setting, the most common indications for prescribing antibiotics during pregnancy are respiratory and urinary tract infections (UTIs). Notably, UTIs are highly prevalent during the second and third trimester (up to $30 \%$ ). ${ }^{45}$ In the postpartum period, antibiotics are also often prescribed to treat infections related to delivery and breast feeding (eg, mastitis). ${ }^{6}$ Several bacterial infections pose a greater risk to mother and child than the antibiotics used to treat the infections. In general, pregnant and breastfeeding women should be treated like other patients; antibiotics should only be prescribed when strictly needed, narrow-spectrum antibiotics should be preferred over broad-spectrum ones, and adherence should be promoted by adequate patient counselling. ${ }^{7-9}$ Nevertheless, the prescription of antibiotics with uncertain safety profile for these patients can be a tradeoff between treating infections and protecting the mother and child against potential side effects. Safety concerns about these antibiotics are based on either theoretical consideration, preclinical findings or conflicting results from epidemiological studies. ${ }^{10}$ Examples include fetal tooth discolouration and inhibition of bone growth caused by tetracyclines, ototoxicity by certain 
aminoglycosides, and concern regarding teratogenicity of fluoroquinolones. Such concerns impact clinical recommendations on how individual groups of antibiotics should be used during and after pregnancy. ${ }^{811}$ Consequently, antibiotic use during pregnancy and breastfeeding period may differ substantially from the period before pregnancy in term of prescribing rate, type of prescribed antibiotics and indication.

Differences in antibiotic fills throughout pregnancy (eg, higher filling rates in late pregnancy) compared with the periods before and after pregnancy also suggest potential impact of pregnancy-related events such as planning or awareness of pregnancy and delivery on antibiotic fills. ${ }^{24}$ Novel techniques of determining and visualising drug utilisation like interrupted time-series analysis (ITSA) using the start of pregnancy or delivery as break point can improve insight on the impact of these events, including both direction and magnitude, on antibiotic prescription fills in pregnant women. ${ }^{12}$ Moreover, women with infections and antibiotic use in a given pregnancy may be more prone to recurrent infections and antibiotic use in a subsequent pregnancy. ${ }^{13}$ To date, no previous study has follow-up of women from the beginning of their reproductive history to assess antibiotic use in a recurrent pregnancy.

Consistently with other countries, antibiotics are commonly prescribed for pregnant and breastfeeding women in Norway. In addition, clinical guidelines of antibiotic use for pregnant and breastfeeding women in primary care have been developed. ${ }^{81114}$ Previous study on medication use in Norwegian pregnant women (20052015) showed that about $28 \%$ of pregnancies in Norway filled antibiotics during their pregnancy in primary care setting. Filling rate during the first trimester $(9.5 \%)$ was lowest among pregnancy-related periods: prepregnancy $(10.2 \%)$, second $(12.2 \%)$ and third $(12.9 \%)$ trimester, and post partum (16.7\%). ${ }^{1}$ However, this descriptive study did not explore various components of antibiotic use (eg, broad-spectrum antibiotic use and adherence to guidelines), factors associated with antibiotic fills in pregnant women and potential drivers of the changes in antibiotic fills throughout pregnancy. Having up to date knowledge about patterns of antibiotic utilisation is essential for public health surveillance, for identifying potential areas of improvement in clinical practice and for promoting judicious prescription of antibiotics among pregnant women.

In the present study, we aim at: (1) estimating changes in antibiotic prescription fills in pregnancy time windows using ITSA, (2) identifying components of antibiotic fills and factors associated with antibiotic fills during pregnancy, and (3) describing the rates of recurrent antibiotic use in subsequent pregnancies.

\section{METHODS}

\section{Data sources}

We conducted a drug utilisation study based on data from 2006 to 2016 from the Medical Birth Registry of
Norway (MBRN) linked to the Norwegian Prescription Database (NorPD) using unique personal identification numbers. ${ }^{15} 16$

The MBRN is a population-based registry containing information on all births in Norway since 1967. MBRN is based on mandatory notification of all pregnancies lasting more than 12 weeks. In MBRN, the information available for each of pregnancy include maternal identification, demographic information, information on the mother's health before and during pregnancy, complications during pregnancy and delivery, date of birth and gestational length and other information on the infants. ${ }^{1617}$

In brief, the NorPD is a nationwide registry on all prescribed medications irrespective of reimbursement, dispensed at pharmacies to individual patients treated in primary care from 1 January 2004. In NorPD, the information available for each dispensed drug is the trade name, pharmaceutical form, strength, package size, number of packages, reimbursement code and dispensing date. No information on the usage is included. The medications are classified according to the Anatomical Therapeutic Chemical (ATC) classification system. ${ }^{15} 18$

\section{Study population}

We included pregnancies having a maternal ID registered in MBRN with valid gestational length (ie, 12 weeks $\leq$ gestational length $\leq 45$ weeks), and child birth year/end of pregnancy between 2006 and 2016 regardless of pregnancy outcomes.

In the analyses of recurrent antibiotic prescription fills in subsequent pregnancies, we restricted to women having their first pregnancy and at least one subsequent pregnancy during the study period.

\section{Antibiotic prescription fills}

We investigated the prescription fills of antibacterials for systemic use (ATC code starting with J01) registered in NorPD. Prescription fills of topical formulations of antibiotics were not included.

\section{Pregnancy-related periods}

We defined the following five pregnancy-related periods: one prepregnancy period up to 3 months before pregnancy; three pregnancy periods, including first trimester (days 1-90 after the start of pregnancy), second trimester (days 91-180), and third trimester (day 180 onwards); and one postpartum period up to 3 months after the end of pregnancy. The first day of the last menstrual period (LMP) is estimated by subtracting the gestational age at delivery from the pregnancy end date. Due date, and thus, gestational length and LMP, is estimated by ultrasound, and only if unavailable, by the women's recall of LMP.

We hypothesised that the recognition of pregnancy preceded changes in antibiotic prescription filling patterns. Consistently with the literature, we assumed early and common pregnancy recognition at fourth and seventh weeks after the start of pregnancy, respectively. ${ }^{12}$ 


\section{Covariates}

We studied maternal sociodemographic factors (including maternal age at delivery, marital status, smoking in pregnancy), factors related to pregnancy or previous pregnancies (including parity, multiple pregnancies, obstetric complications during pregnancy, asthma, recurrent UTI, smoking in pregnancy and previous pregnancy loss), and comedication. Comedication included fertility treatment using clomiphene (ie, ATC code G03GB02) before pregnancy as marker of infertility, use of folic acid before pregnancy as marker of pregnancy planning, medications used for musculoskeletal (ie, ATC M) and nervous system (ie, ATC N)) as these drugs can be used to relieve symptoms associated with infections. Furthermore, drugs from ATC $\mathrm{N}$ can be used in patients with psychiatric disorders whose prevalence of infections is higher than the general population. Obstetric complications were measured by obstetric comorbidity index adapted from Bateman et al. ${ }^{19}$

\section{Measures of antibiotic use}

The main outcome measure was the proportion of exposed pregnancies aggregated by week in pregnancy episode (ie, number of exposed pregnancies per 100 pregnancies). Exposed pregnancies were pregnancies with at least one antibiotic prescription filled in a specific pregnancy-related period based on the date of dispensing.

The secondary outcome measures included (1) the proportion of broad-spectrum antibiotic prescriptions and (2) the proportion of first line antibiotic prescriptions among all antibiotic prescriptions in a specific period. Broad-spectrum antibiotics were defined as per European Surveillance of Antimicrobial Consumption Network's definition. ${ }^{20}$ First-line antibiotics were defined using clinical guidelines of antibiotic use for pregnant and breastfeeding women in primary care in Norway (online supplemental eTable 1 ). ${ }^{8}$

The outcome measure of recurrent use was the proportion of women who filled antibiotic prescription in a subsequent pregnancy.

\section{Data analyses}

First, proportion of exposed pregnancies (in overall and by therapeutic classes) and proportion of broad-spectrum antibiotic and first-line antibiotic prescription filled were calculated for different pregnancy-related periods.

Second, we described maternal characteristics and comedications of pregnant women with and without antibiotic prescription fills and explored the extent and patterns of missing data on covariates of interest.

Third, the association between antibiotic prescription filled (dichotomised as yes/no) during pregnancy and the above-mentioned covariates was investigated using generalised estimating equations (GEE) for Poisson regression, limited to pregnancies without missing data on these covariates. This approach made it possible to handle correlated data due to repeated participation of some women in the cohort. Because of explanatory purpose, we included all covariates in the final multivariable model. Adjusted estimates were expressed as ORs with corresponding 95\% CIs.

Fourthly, we assessed changes in the proportion of exposed pregnancies aggregated by week using the ITSA with two break points (week 4 and week 7) using data spanning from week 12 before the start of pregnancy to week 42 thereafter. The ITSA was based on the segmented linear regression modelling (detailed modelling strategies in online supplemental appendix). ${ }^{2122}$ We repeated ITSA with one interruption (end of pregnancy) to assess the changes in proportion of exposed pregnancies aggregated by week following the end of pregnancy (data spanning from week 42 before the end of pregnancy to week 12 afterwards).

Lastly, the proportion of women filling antibiotics, stratified by whether antibiotics were filled in the previous pregnancy, were calculated (results were presented until the fourth pregnancy).

\section{Additional analyses}

Sensitivity analysis using normal logistic regression was performed to check the robustness of findings from GEE model.

The use of folic acid before pregnancy is a potential marker of pregnancy planning which may lead to changes in willingness to fill antibiotic prescriptions. ${ }^{23}$ In Norway, this information is routinely recorded in MBRN. Nearly one-third of general birthing population in Norway report folic acid use prior to pregnancy. ${ }^{24}{ }^{25}$ We performed additional ITSA among those with and without acid folic use before pregnancy to assess the potential role of pregnancy planning on antibiotic prescription fills.

We also applied ITSA: (1) for medications used for musculoskeletal and nervous system to contrast the changes detected by ITSA for antibiotics and (2) for main antibiotic therapeutic classes.

Data management and statistical analyses were performed with Stata/MP V.16.0 for Windows.

\section{RESULTS}

\section{Antibiotic fills before, during and after pregnancy}

The study population consisted of 653058 pregnancies among 423 604women having valid maternal ID with gestational length between 12 and 45 weeks ended in 2006-2016 (figure 1).

A total of 180516 out of 653058 pregnancies filled at least one antibiotic prescription during pregnancy $(27.6 \%)$ and the prescription filling rates decreased over the study period (online supplemental eTable 2 and eFigure 1). The proportion of pregnancies exposed to antibiotics in the 3 months before pregnancy, first trimester, second trimester, third trimester and 3 months after pregnancy were $10.9 \%, 9.9 \%, 11.3 \%, 13.2 \%$ and $16.1 \%$ respectively. Penicillins (J01C) remained the most common antibiotic class filled in any pregnancy periods $(>60 \%)$ and were increasingly filled during pregnancy and after pregnancy. The top 10 filled antibiotics were 


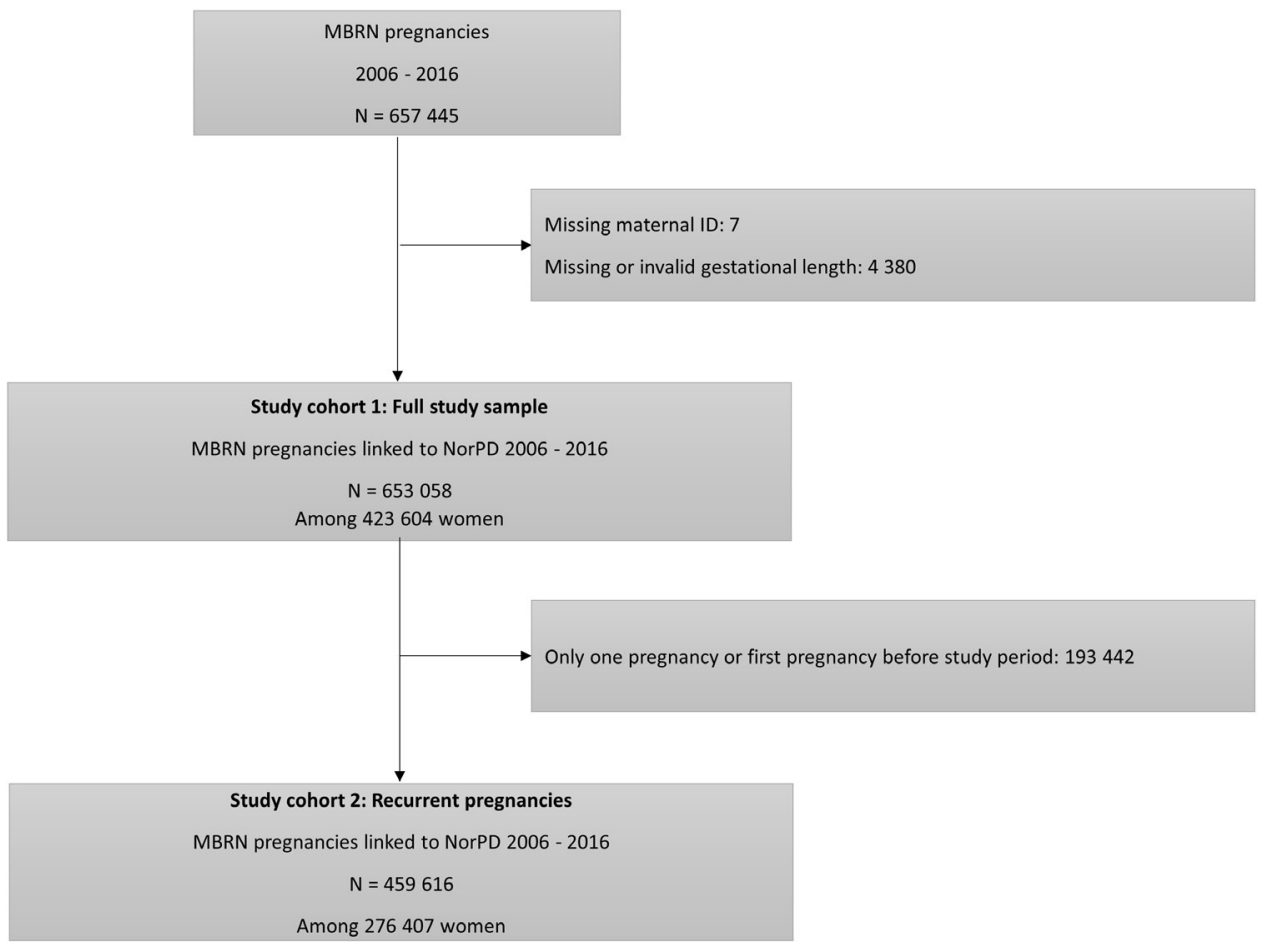

Figure 1 Flow chart of the study population (2006-2016). MBRN, Medical Birth Registry of Norway.

presented in online supplemental eTable 3. There were more first-line and less broad-spectrum antibiotics filled during pregnancy than before and after pregnancy (online supplemental eTable 4).

\section{Characteristics of antibiotic prescription fills during pregnancy}

Table 1 described the characteristics of pregnancies with and without antibiotic prescription fills.

The GEE modelling, performed on 653046 pregnancies with complete data on all covariates (12 pregnancies excluded because of missing marital status, the only covariate with missing data), revealed increased risk of filling an antibiotic during pregnancy among women with a history of recurrent UTIs (adjusted OR=2.65, 95\% CI 2.59 to 2.72), comedication with drugs used for musculoskeletal (adjusted $\mathrm{OR}=1.55,95 \% \mathrm{CI} 1.50$ to 1.60 ) and nervous system (adjusted OR=1.83, 95\% CI 1.80 to 1.87 ), low maternal age (adjusted $\mathrm{OR}=1.39$, 95\% CI 1.37 to 1.42), and other factors (table 1 ). Sensitivity analysis using logistic regression yielded consistent findings (results not shown).

\section{Changes in antibiotic filling following the recognition or end of pregnancy}

Before the fourth week into pregnancy, the proportion of pregnancies exposed to antibiotic decreased (figure 2A). ITSA showed an immediate change of -0.07 percentage points $(95 \%$ CI -0.13 to -0.01 ) in the proportion of exposed pregncies after this break point while no change in slope was observed. After the seventh week into pregnancy, both level and slope increased but we could not reject the null (online supplemental eTable 5). In the period after the potential recognition of pregnancy (week 4 to week 7 into pregnancy), the proportion of pregnancies exposed to antibiotics gradually increased by 0.01 percentage points/week (95\% CI 0.01 to 0.02 ).

Before the delivery, the proportion of pregnancies exposed to antibiotics remained stable (online supplemental eTable 6 and figure 2B). The proportion increased by 1.61 percentage point (95\% CI 0.31 to 2.91 ) immediately after delivery while the slope decreased remarkably afterwards (change in slope $=-0.19$ percentage points, $95 \%$ CI -0.34 to -0.05)) (online supplemental eTable 6).

\section{Additional analyses}

Among women using folic acid prior to pregnancy, proportion of pregnancies exposed to antibiotics dropped by 0.11 percentage points $(95 \% \mathrm{CI}-0.14$ to -0.08$)$ ) after the 4 weeks into pregnancy breaking point, remained stable between week 4 and week 7 into pregnancy then increased by 0.01 percentage points/week (95\% CI 0.01 to 0.02 ) afterwards (online supplemental eTable 5 and eFigure 2). Among women without prior folic acid use, no major change was detected after the recognition of pregnancy. Yet the proportion increased during the recognition window by 0.02 percentage point/week ( $95 \%$ CI 0.01 to 0.02 ) (online supplemental eTable 5 and eFigure 2).

The proportion of pregnancies exposed to medications used for musculoskeletal systems dropped by 0.12 percentage points/week during the recognition window (95\% CI -0.12 to-0.11). This proportion remained low during the remaining duration of pregnancy (online supplemental eFigure 3). After delivery, the proportion rose steeply (immediate change $=0.5$ percentage points, 
Table 1 Characteristics of pregnancies with and without antibiotic prescription fills and factors associated with antibiotic prescription fills, Norway, 2006-2016, 653058 pregnancies

\begin{tabular}{|c|c|c|c|c|c|}
\hline \multirow[b]{2}{*}{ Characteristic } & \multirow[b]{2}{*}{$\begin{array}{l}\text { Study population } \\
\mathbf{N}(\%)\end{array}$} & \multirow[b]{2}{*}{$\begin{array}{l}\text { No antibiotic } \\
\text { prescription filled } \\
\text { N (\%) }\end{array}$} & \multirow[b]{2}{*}{$\begin{array}{l}\text { Any antibiotic } \\
\text { prescription filled } \\
\mathbf{N}(\%)\end{array}$} & \multicolumn{2}{|c|}{ Generalised estimating equations } \\
\hline & & & & $\begin{array}{l}\text { Univariable } \\
\text { OR } \\
(95 \% \mathrm{Cl})\end{array}$ & $\begin{array}{l}\text { Multivariable } \\
\text { Adjusted OR } \\
(95 \% \mathrm{Cl})\end{array}$ \\
\hline \multicolumn{6}{|l|}{ Maternal age } \\
\hline$\leq 24$ years & 102308 (15.7) & 66916 (14.2) & $35392(19.6)$ & 1.37 (1.35 to 1.40$)$ & 1.39 (1.37 to 1.42$)$ \\
\hline 25-29 years & 206141 (31.6) & 149768 (31.7) & $56373(31.2)$ & 1.00 & 1.00 \\
\hline $30-34$ years & 216922 (33.2) & 161266 (34.1) & $55656(30.8)$ & 0.93 (0.91 to 0.94$)$ & 0.89 (0.87 to 0.90$)$ \\
\hline$\geq 35$ years & 127687 (19.5) & $94592(20.0)$ & 33095 (18.3) & 0.93 (0.92 to 0.95$)$ & 0.77 (0.76 to 0.79$)$ \\
\hline \multicolumn{6}{|l|}{ Marital status } \\
\hline Married/cohabiting & 604916 (92.6) & $441287(93.4)$ & 163629 (90.6) & 1.00 & 1.00 \\
\hline Other & $48130(7.4)$ & $31248(6.6)$ & $16882(9.4)$ & 1.42 (1.39 to 1.48$)$ & 1.29 (1.26 to 1.31$)$ \\
\hline \multicolumn{6}{|l|}{ Parity } \\
\hline 0 & 267432 (42.3) & 204627 (43.3) & 71805 (39.8) & 1.00 & 1.00 \\
\hline 1 & $376626(57.7)$ & 267915 (56.7) & 108711 (60.2) & 1.15 (1.14 to 1.17$)$ & 1.30 (1.29 to 1.32$)$ \\
\hline \multicolumn{6}{|l|}{ Plurality } \\
\hline Singleton & $641972(98.3)$ & $464500(98.3)$ & 177472 (98.3) & 1.00 & 1.00 \\
\hline Multiple & $11086(1.7)$ & $8042(1.7)$ & 3044 (1.7) & 0.99 (0.95 to 1.03$)$ & 0.93 (0.89 to 0.97$)$ \\
\hline \multicolumn{6}{|l|}{ Obstetric index* } \\
\hline 0 & 430615 (65.9) & 313376 (66.3) & 117239 (64.9) & 1.00 & 1.00 \\
\hline 1 & $146744(22.5)$ & 105435 (22.3) & 41309 (22.9) & 1.05 (1.03 to 1.06$)$ & 1.09 (1.07 to 1.11$)$ \\
\hline$\geq 2$ & 75699 (11.6) & 53731 (11.4) & 21968 (12.2) & 1.09 (1.07 to 1.11$)$ & 1.17 (1.14 to 1.20$)$ \\
\hline \multicolumn{6}{|c|}{ Previous miscarriage or stillbirth } \\
\hline Yes & $185847(28.5)$ & $132408(28.0)$ & $53439(29.6)$ & 1.07 (1.06 to 1.09$)$ & 1.05 (1.04 to 1.06$)$ \\
\hline No & $467211(71.5)$ & $340134(72.0)$ & $127077(70.4)$ & 1.00 & 1.00 \\
\hline \multicolumn{6}{|l|}{ Asthma } \\
\hline Yes & $30307(4.6)$ & $19505(4.1)$ & $10802(6.0)$ & 1.46 (1.42 to 1.49$)$ & 1.24 (1.21 to 1.28$)$ \\
\hline No & $622751(95.4)$ & 453037 (95.9) & $169714(94.0)$ & 1.00 & 1.00 \\
\hline \multicolumn{6}{|c|}{ Recurrent urinary tract infections } \\
\hline Yes & $26448(4.1)$ & $13212(2.8)$ & $13276(7.4)$ & 2.65 (2.59 to 2.72 ) & 2.62 (2.56 to 2.69 ) \\
\hline No & $626570(95.9)$ & $459330(97.2)$ & $167240(92.6)$ & 1.00 & 1.00 \\
\hline \multicolumn{6}{|l|}{ Smoking in pregnancy } \\
\hline Yes & $116510(17.8)$ & $85406(18.1)$ & $31104(17.2)$ & 0.97 (0.96 to 0.98$)$ & $0.98(0.97$ to 0.99$)$ \\
\hline No & 536548 (82.2) & $387136(81.9)$ & $149412(82.8)$ & 1.00 & 1.00 \\
\hline \multicolumn{6}{|c|}{ Folic acid before pregnancy } \\
\hline Yes & $183898(28.2)$ & $137241(29.0)$ & $46657(25.9)$ & 1.00 & 1.00 \\
\hline No & $469160(71.8)$ & 335301 (71.0) & $133859(74.1)$ & $1.16(1.14$ to 1.17$)$ & $1.10(1.08$ to 1.11$)$ \\
\hline \multicolumn{6}{|c|}{ Clomiphene 12 months>pregnancy } \\
\hline Yes & $19654(3.0)$ & $14216(3.0)$ & $5438(3.0)$ & 0.99 (0.96 to 1.02$)$ & $1.10(1.06$ to 1.14$)$ \\
\hline No & $633404(97.0)$ & $458326(96.7)$ & $175078(96.7)$ & 1.00 & 1.00 \\
\hline \multicolumn{6}{|c|}{ Comedication, musculo-skeletal drugs } \\
\hline Yes & $15557(2.4)$ & $8888(1.9)$ & $6669(3.7)$ & 1.91 (1.85 to 1.97$)$ & 1.55 (1.50 to 1.60$)$ \\
\hline No & $637501(97.6)$ & $463654(98.1)$ & 173847 (96.3) & 1.00 & 1.00 \\
\hline \multicolumn{6}{|c|}{ Comedication, nervous system drugs } \\
\hline Yes & $56414(8.6)$ & $32719(6.9)$ & $23695(13.1)$ & 1.97 (1.93 to 2.00$)$ & $1.83(1.80$ to 1.87$)$ \\
\hline
\end{tabular}


Table 1 Continued

\begin{tabular}{|c|c|c|c|c|c|}
\hline \multirow[b]{2}{*}{ Characteristic } & \multirow[b]{2}{*}{$\begin{array}{l}\text { Study population } \\
\mathbf{N}(\%)\end{array}$} & \multirow[b]{2}{*}{$\begin{array}{l}\text { No antibiotic } \\
\text { prescription filled } \\
\mathbf{N}(\%)\end{array}$} & \multirow[b]{2}{*}{$\begin{array}{l}\text { Any antibiotic } \\
\text { prescription filled } \\
\mathbf{N}(\%)\end{array}$} & \multicolumn{2}{|c|}{ Generalised estimating equations } \\
\hline & & & & $\begin{array}{l}\text { Univariable } \\
\text { OR } \\
(95 \% \mathrm{Cl})\end{array}$ & $\begin{array}{l}\text { Multivariable } \\
\text { Adjusted OR } \\
(95 \% \mathrm{Cl})\end{array}$ \\
\hline No & $596644(91.4)$ & $439823(93.1)$ & $156821(86.9)$ & 1.00 & 1.00 \\
\hline
\end{tabular}

Missing numbers: marital status, $\mathrm{n}=12$.

*Adapted from Bateman et al, using the variables available in MBRN (age, asthma, pregestational diabetes, chronic hypertension, kidney disease, previous caesarean section, multiple gestation, severe preeclampsia, mild preeclampsia, gestational hypertension) and weighting the variables as done by Bateman et al.

†Univariable and multivariable regressions were performed on 653046 pregnancies with complete information on all covariates.

$95 \%$ CI 0.15 to 0.87 ) and then dropped to the levels observed before pregnancy. The proportion of pregnancies exposed to medications used for nervous systems followed the similar movement (online supplemental eFigure 3).

Stratified analyses by therapeutics class showed different patterns. Notably, tetracyclines followed the patterns of comedications while the proportion of pregnancies exposed to penicillins increased throughout the pregnancy (online supplemental eFigures 4 and 5).

\section{Recurrent use of antibiotic in subsequent pregnancies}

The assessment of recurrent antibiotic prescription filling revealed that women who had filled antibiotic prescription in a given pregnancy were 1.7 to 3.1 times more likely to fill an antibiotic prescription again in their subsequent pregnancy (figure 3). Notably, among women who had filled antibiotics in all three first pregnancies, 58.7\% filled an antibiotic in their fourth pregnancy compared with $18.9 \%$ among women without history of antibiotic prescription fills during pregnancy.

\section{DISCUSSION}

This study gives an updated nationwide overview of antibiotic prescription fills in pregnant and breastfeeding women in Norway. First, we assessed the antibiotic prescription filling patterns before, during, and after

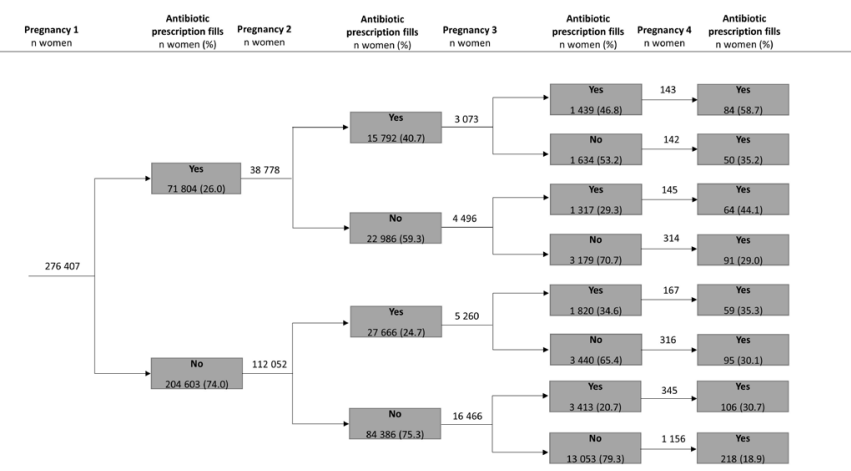

Figure 2 Recurrent antibiotic fills in subsequent pregnancies, 459616 pregnancies among 276407 women with their first pregnancy (irrespective of outcome) in the study period (2006-2016) having at least one subsequent pregnancy. pregnancy and took a step further to determine factors associated with antibiotic prescription fills as well as filling patterns of first-line and broad-spectrum antibiotics. Second, our study confirmed the strong impact of pregnancy, especially pregnancy planning, and delivery

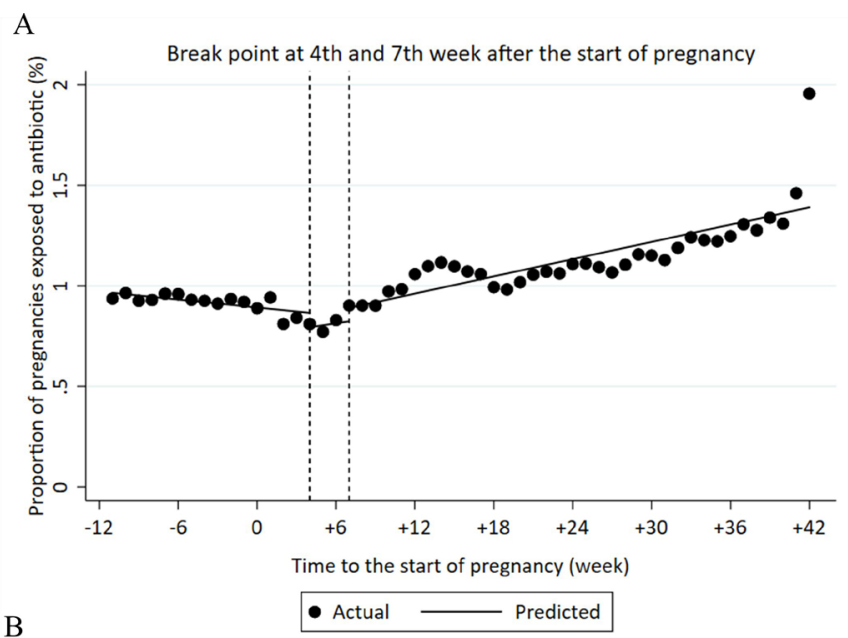

B

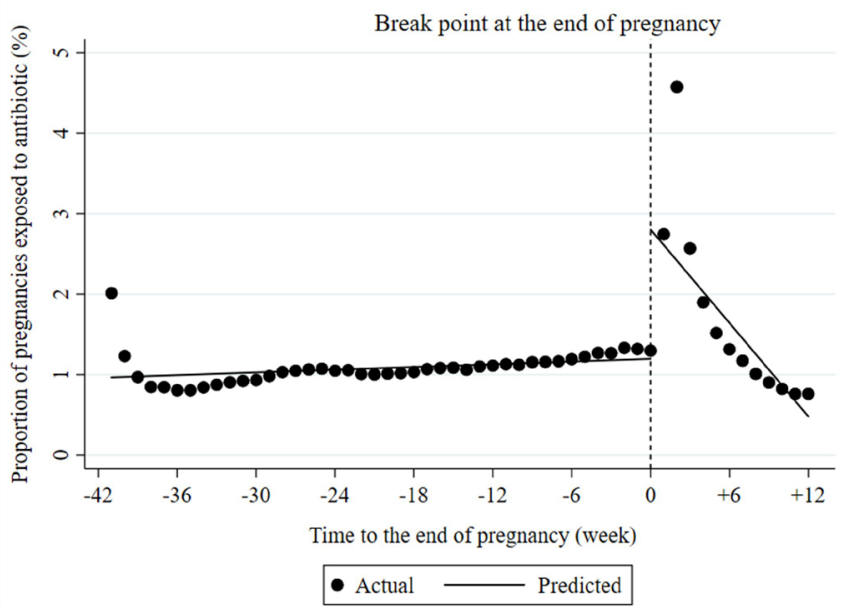

Figure 3 Interrupted time series analysis: (A) proportion of pregnancies exposed to antibiotics in each of the 12 weeks before and 24 weeks after the start of pregnancy with break points at the fourth and seventh week after the start of pregnancy, (B) proportion of pregnancies exposed to antibiotics in each of the 24 weeks before and 12 weeks after delivery/end of pregnancy with break point at delivery/end of pregnancy. 
on proportion of pregnancies exposed to antibiotics and other medications using ITSA-a powerful quasiexperimental method. Third, we brought an attention to a novel aspect of antibiotic utilisation-the recurrent antibiotic fills patterns in pregnant women. These highlighted findings could assist with better dissemination and implementation of future strategies to promote judicious use of antibiotics in pregnancy.

This study also shows the advantages of using modern analytical methods in perinatal drug utilisation studies. ITSA, a modelling strategy to estimate changes following interventions, is widely used to assess impact of policy changes or guidelines on drug utilisation at the national level. ${ }^{21} 2226$ The implementation of this method using pregnancy-related events as breakpoints, is particularly useful to visualise and study the effects of pregnancy and delivery on medication filling patterns, yet uptake of this method has been slow in perinatal pharmacoepidemiology. We have been able to identify only one prior study using this method to characterise drug utilisation in pregnancy. ${ }^{12}$ This is unfortunate, because of the potential advantages and insights this method might bring to the field.

Over a fourth of pregnancies in Norway filled antibiotic, consistently previous literature. ${ }^{1}$ Compared with other nationwide studies estimating the proportion of pregnant women who filled antibiotics among all pregnant women, the filling rate of Norway might be lower than those of Denmark (30\%-37\%) and UK (34\%) but higher than those of Germany $(19.7 \%)$ and Netherlands $(20.8 \%) .^{2927} 28$ The differences in clinical guidelines and prescribing routine between Norway and other countries may explain these variations. ${ }^{29}$ Interestingly, the filling rates in pregnant women do not seem to mirror antibiotic consumption in general population with Denmark and Norway sharing the same rates while Netherlands was the lowest consumer. ${ }^{30}$

During pregnancy, the proportion of exposed pregnancies was found to be lowest in the first trimester. This probably reflects the impact of pregnancy recognition because we also detected a sudden drop in the proportion of exposed pregnancies after the period spanning from 4 to 7 weeks into pregnancy using the ITSA. Higher proportion of exposed pregnancies observed later in pregnancy could be explained by screening, detecting and treating asymptomatic bacteriuria and UTIs in pregnancy, as recommended in routine maternity care. ${ }^{5} 31$ This is supported by our finding that having recurrent UTIs was associated with almost a threefold higher likelihood of antibiotic prescription fills compared with women without recurrent UTIs. The proportion of exposed pregnancies in the 3 months after pregnancy was highest among all pregnancy-related periods, potentially related to mastitis that occurs in up to $10 \%$ of breastfeeding women and infections associated with delivery. ${ }^{6}{ }^{32}$ Similar patterns were observed in the Netherlands and in Denmark. ${ }^{13} 28$

We noted in our study a shift towards first-line antibiotics (nearly $90 \%$ during pregnancy compared with $60 \%$ before pregnancy) suggesting high awareness and compliance of physicians to clinical guidelines of antibiotic use in pregnancy. Previous studies showed similar findings with more antibiotics with better safety profiles prescribed for pregnant women. ${ }^{28}$ The exposure to second-line and third-line antibiotics during pregnancy, which was very limited, could be explained by unrecognised pregnancy or severe infections where the benefits of the antibiotic use outweigh the risks. Importantly, the proportion of pregnancies exposed to tetracyclines, which are contraindicated during the second and third trimester, were less than $0.1 \%$. Besides, the proportion of broad-spectrum antibiotic fills before pregnancy was found to be much lower compared with the rest of Europe and North America. ${ }^{29}{ }^{33}$ Broad-spectrum antibiotics were even less prescribed during and after pregnancy. This could be explained by a high focus on using narrow-spectrum antibiotics as first-line therapy and low resistance rate to these antibiotics in Norway. ${ }^{34}$

The drop in the proportion of exposed pregnancies detected around the 4-7 weeks into pregnancy (ie, potential pregnancy recognition window) by ITSA could explain the low proportion of exposed pregnancies in the first trimester. Notably, the change was more visible among those who potentially planned their pregnancy (ie, reported folic acid use before pregnancy). However, the impact of pregnancy recognition seems to be modest on antibiotics compared with other medications (including medications used for musculoskeletal and nervous systems, antidepressants and psychostimulants). ${ }^{12}$ Notably, after the recognition window, the proportion of pregnancies exposed to antibiotic slowly increased while the proportion of pregnancies exposed to other medications remained low throughout the pregnancy. This gradual increase observed particularly for antibiotics is likely to be driven by the detection of infections during maternity care checkups (first visit recommended between weeks 6 and 10 in Norway) and an increased willingness to prescribe medications after the first trimester (ie, organogenesis) ${ }^{35}$ Similarly, the sudden rise detected by ITSA after delivery was the main driver for peak observed in the 3 months after pregnancy. The peak observed shortly after birth could be explained by clinical need to treat or to prevent infections arising from delivery-related wound care and breast feeding. ${ }^{6}$ Indeed, the proportion of exposed pregnancies was not high throughout the whole period as seen in the ITSA's visualisation.

Interestingly, we found that women who filled antibiotic in a pregnancy were up to three times more likely to fill antibiotic in a subsequent pregnancy, indicating that antibiotic prescription patterns from one pregnancy is carried over to the next. This could be indicative of a group of women more prone to infections and/or are more willing to use antibiotics during pregnancy. ${ }^{13}$ Studies have demonstrated that history of previous infections during pregnancy (notably UTIs) highly increased the likelihood of recurrent infections in subsequent 
pregnancies. ${ }^{36}{ }^{37}$ Women successfully treated with antibiotics delivering a healthy child may be more willing to adhere to prescribed antibiotics in a subsequent pregnancy compared with women with no prior experience with antibiotic use in pregnancy. ${ }^{38}$

\section{Strengths and limitations}

To our knowledge, this is the first study that employs ITSA to examine the effect of pregnancy-related events on antibiotic prescription filling patterns. We believe that this method could be highly beneficial for researchers in perinatal pharmacoepidemiology, and could be applied to all therapeutic areas. Moreover, we are among the first to follow-up women from the beginning of their reproductive history to quantify recurrent antibiotic fills in subsequent pregnancies. This study was conducted on prospectively collected information obtained from linked electronic healthcare registries covering an entire nation. However, this study has some limitations. First, our study did not capture pregnancies lasting less than 12 weeks (spontaneous and induced abortions) as these pregnancies are not recorded in MBRN. The patterns of antibiotic prescription fills of these pregnancies might be different from those of pregnancies included in our study population. Second, our study did not capture antimycotics or other formulations than oral formulations despite that they are often used among women with infections in pregnancy. For example, oral metronidazole which can be prescribed for treating symptomatic bacterial vaginosis was not included as it is not classified under ATC J01. Third, we did not have access to information about indications. Therefore, it is difficult to determine whether a prescription was appropriate or not. Fourth, the filling rate may not truly reflect the prescribing rate. Indeed, in a study based on visits to 458 general practitioners, Fossum et al estimated that $83 \%$ of antibiotic prescriptions for pregnant women were filled in the pharmacy. ${ }^{39} \mathrm{Fifth}$, we do not know if the filled antibiotic prescriptions were taken by the pregnant women. As a result, the filling rate may overestimate the actual utilisation. Sixth, information regarding breastfeeding is not recorded in MBRN. Breastfeeding mothers may have different antibiotic filling patterns compared with non-breastfeeding ones. ${ }^{40}$ Seventh, the ITSA is performed with an assumption that no other event than the interruption could have impact on the outcome measure. For antibiotics, this is far from the reality. Of note, the assumed interruption points at gestational weeks 4 and 7 are serving as estimates rather than cut-points when a pregnancy may be identified. Therefore, the outputs from ITSA must be interpreted with caution. Last but not least, because we included all pregnancies in the ITSA regardless of calendar year of delivery, temporal changes in antibiotic prescription fills during pregnancy were not captured in our study. Overall antibiotic prescription fills (notably macrolides) during pregnancy seems to declining in the recent years, possibly as a result of large-scale effort to improve antibiotic prescribing.

\section{CONCLUSION}

ITSA approach visualised and highlighted the important impact of pregnancy and delivery on antibiotic prescription filling patterns. This method is a promising analytic tool for perinatal pharmacoepidemiology. Women who filled antibiotic in a pregnancy were more likely to fill antibiotic again in the subsequent pregnancy. This evidence might be helpful for prescribers of antibiotics in pregnancy and healthcare professionals caring for pregnant women in clinical practice.

Twitter Nhung Thi Hong Trinh @NhungPharma and Hedvig Marie Egeland Nordeng @Pharma_Nordeng

Acknowledgements Data were stored at the TSD (Tjeneste for Sensitive Data) facilities, owned by the University of Oslo, operated and developed by the TSD service group at the University of Oslo, IT-Department (USIT) (tsd-drift@usit.uio.no).

Contributors NTHT: design of study, data management, data analysis, drafting of the manuscript, final approval of the version to be published and guarantor. $\mathrm{SH}$ : design of study, data management, revising of the manuscript, final approval of the version to be published. HMEN: conception of study, design of study, revising of the manuscript, final approval of the version to be published.

Funding NTHT was supported by a PharmaTox QLS Visiting Scholarship. HME Nordeng and Sarah Hjorth are supported by a European Research Council Starting Grant Drugs In Pregnancy (grant number 639377).

\section{Competing interests None declared.}

Patient consent for publication Not applicable.

Ethics approval The study was approved by the Regional Committee for Research Ethics in South Eastern Norway (approval number 2018/140/REK Sør Øst) and by the Data Protection Officer at the University of Oslo (approval number 58033). Data were handled in accordance with the General Data Protection Regulation.

Provenance and peer review Not commissioned; externally peer reviewed.

Data availability statement Data may be obtained from a third party and are not publicly available. The data in this project were delivered by the registry holders to the researchers as pseudonymised data files. Data are available upon request to the registry holders, provided legal and ethical approvals.

Supplemental material This content has been supplied by the author(s). It has not been vetted by BMJ Publishing Group Limited (BMJ) and may not have been peer-reviewed. Any opinions or recommendations discussed are solely those of the author(s) and are not endorsed by BMJ. BMJ disclaims all liability and responsibility arising from any reliance placed on the content. Where the content includes any translated material, BMJ does not warrant the accuracy and reliability of the translations (including but not limited to local regulations, clinical guidelines, terminology, drug names and drug dosages), and is not responsible for any error and/or omissions arising from translation and adaptation or otherwise.

Open access This is an open access article distributed in accordance with the Creative Commons Attribution Non Commercial (CC BY-NC 4.0) license, which permits others to distribute, remix, adapt, build upon this work non-commercially, and license their derivative works on different terms, provided the original work is properly cited, appropriate credit is given, any changes made indicated, and the use is non-commercial. See: http://creativecommons.org/licenses/by-nc/4.0/.

\section{ORCID iDs}

Nhung Thi Hong Trinh http://orcid.org/0000-0003-2794-5322

Sarah Hjorth http://orcid.org/0000-0003-2841-5868

\section{REFERENCES}

1 Engeland A, Bjørge T, Klungsøyr K, et al. Trends in prescription drug use during pregnancy and postpartum in Norway, 2005 to 2015. Pharmacoepidemiol Drug Saf 2018;27:995-1004.

2 Amann U, Egen-Lappe V, Strunz-Lehner C, et al. Antibiotics in pregnancy: analysis of potential risks and determinants in a large German statutory sickness fund population. Pharmacoepidemiol Drug Saf 2006;15:327-37. 
3 Olesen C, Steffensen FH, Nielsen GL, et al. Drug use in first pregnancy and lactation: a population-based survey among Danish women. The EUROMAP group. Eur J Clin Pharmacol 1999;55:139-44.

4 Petersen I, Gilbert R, Evans S, et al. Oral antibiotic prescribing during pregnancy in primary care: UK population-based study. J Antimicrob Chemother 2010;65:2238-46.

5 Glaser AP, Schaeffer AJ. Urinary tract infection and bacteriuria in pregnancy. Urol Clin North Am 2015;42:547-60.

6 Chaim W, Burstein E. Postpartum infection treatments: a review. Expert Opin Pharmacother 2003;4:1297-313.

7 Ministry of Health and Care Services. [National strategy against antibiotic resistance 2015-2020]. Available: https://www.regjeringen. no

8 Nordeng $\mathrm{H}$. [Antibiotic use in the primary health care service for pregnant and breastfeeding women]. Available: http://www.antibiot ikaiallmennpraksis.no

9 National Institute for Health and Care Excellence. Summary of antimicrobial prescribing guidance - managing common infections. Available: https://www.nice.org.uk/Media/Default/About/what-we-do/ NICE-guidance/antimicrobial\%20guidance/summary-antimicrobialprescribing-guidance.pdf

10 Niebyl JR. Antibiotics and other anti-infective agents in pregnancy and lactation. Am J Perinatol 2003;20:405-14.

11 Nordeng S, Nordeng H, Høye S. [Use of antibiotics during pregnancy]. Tidsskr Nor Laegeforening 2016;136:317-21.

12 Cohen JM, Selmer R, Furu K, et al. Interrupted time series analysis to assess changes in prescription filling around conception and implications for exposure misclassification. Pharmacoepidemiol Drug Saf 2020;29:745-9.

13 Stokholm J, Schjørring S, Pedersen L, et al. Prevalence and predictors of antibiotic administration during pregnancy and birth. PLoS One 2013;8:e82932.

14 NICE UK. Guidances on antimicrobial stewardship. Available: https:// www.nice.org.uk

15 Furu K, Strøm H, Rønning M. The Norwegian prescription database (NorPD): new register for pharmacoepidemiological research covering a whole nation. Pharmacoepidemiol Drug Saf 2005; $14:$ :S48.

16 Irgens LM. The medical birth registry of Norway. epidemiological research and surveillance throughout 30 years. Acta Obstet Gynecol Scand 2000;79:435-9.

17 Langhoff-Roos J, Krebs L, Klungsøyr K, et al. The Nordic medical birth registers---a potential goldmine for clinical research. Acta Obstet Gynecol Scand 2014;93:132-7.

18 Furu K, Wettermark B, Andersen M, et al. The Nordic countries as a cohort for pharmacoepidemiological research. Basic Clin Pharmacol Toxicol 2010;106:86-94.

19 Bateman BT, Mhyre JM, Hernandez-Diaz S, et al. Development of a comorbidity index for use in obstetric patients. Obstet Gynecol 2013;122:957-65.

20 Coenen S, Ferech M, Haaijer-Ruskamp FM, et al. European surveillance of antimicrobial consumption (ESAC): quality indicators for outpatient antibiotic use in Europe. Qual Saf Health Care 2007;16:440-5.
21 Bernal JL, Cummins S, Gasparrini A. Interrupted time series regression for the evaluation of public health interventions: a tutorial. Int J Epidemiol 2017;46:348-55.

22 Jandoc R, Burden AM, Mamdani M, et al. Interrupted time series analysis in drug utilization research is increasing: systematic review and recommendations. J Clin Epidemiol 2015;68:950-6.

23 Stern J, Salih Joelsson L, Tydén T, et al. Is pregnancy planning associated with background characteristics and pregnancy-planning behavior? Acta Obstet Gynecol Scand 2016;95:182-9.

24 Kinnunen TI, Sletner L, Sommer C, et al. Ethnic differences in folic acid supplement use in a population-based cohort of pregnant women in Norway. BMC Pregnancy Childbirth 2017;17:143.

25 Nilsen RM, Mastroiacovo P, Gunnes N, et al. Folic acid supplementation and interpregnancy interval. Paediatr Perinat Epidemiol 2014:28:270-4.

26 Trinh NTH, Bruckner TA, Lemaitre M, et al. Association between national treatment guidelines for upper respiratory tract infections and outpatient pediatric antibiotic use in France: an interrupted timeseries analysis. J Pediatr 2020;216:88-94.

27 Dixon JM. Lactational mastitis. Available: https://www.uptodate.com/ contents/lactational-mastitis

28 de Jonge L, Bos HJ, van Langen IM, et al. Antibiotics prescribed before, during and after pregnancy in the Netherlands: a drug utilization study. Pharmacoepidemiol Drug Saf 2014;23:60-8.

29 Borg MA, Camilleri L. Broad-Spectrum antibiotic use in Europe: more evidence of cultural influences on prescribing behaviour. $J$ Antimicrob Chemother 2019;74:3379-83.

30 Adriaenssens N, Coenen S, Versporten A, et al. European surveillance of antimicrobial consumption (ESAC): outpatient antibiotic use in Europe (1997-2009). J Antimicrob Chemother 2011;66 Suppl 6:vi3-12.

31 Cunningham FG, Lucas MJ. Urinary tract infections complicating pregnancy. Baillieres Clin Obstet Gynaecol 1994;8:353-73.

32 Nordeng H, Tufte E, Nylander G. [Treatment of mastitis in general practice]. Tidsskr Nor Laegeforen 2003;123:3027-30.

33 King LM, Bartoces M, Fleming-Dutra KE, et al. Changes in US outpatient antibiotic prescriptions from 2011-2016. Clin Infect Dis 2020;70:370-7.

34 Simonsen GS. [Surveillance and prevalence of antimicrobial resistance in Norway]. Tidsskr Nor Laegeforen 2009;129:623-7.

35 Overview of recommendations in maternity care. Available: https:// www.helsedirektoratet.no

36 McCormick T, Ashe RG, Kearney PM. Urinary tract infection in pregnancy. Obstet Gynaecol 2008;10:156-62.

37 Le J, Briggs GG, McKeown A, et al. Urinary tract infections during pregnancy. Ann Pharmacother 2004;38:1692-701.

38 Petersen I, McCrea RL, Lupattelli A, et al. Women's perception of risks of adverse fetal pregnancy outcomes: a large-scale multinational survey. BMJ Open 2015;5:e007390.

39 Fossum GH, Gjelstad S, Kværner KJ, et al. Prescribing antibiotics when the stakes are higher - do GPs prescribe less when patients are pregnant? A retrospective observational study. BJGP Open 2018;2:bjgpopen18X101505.

40 de Sá Del Fiol F, Barberato-Filho S, de Cássia Bergamaschi C, et al. Antibiotics and breastfeeding. Chemotherapy 2016;61:134-43. 